\title{
Lophophorates prove likewise variable
}

Evidence from molecular phylogeny challenges the conventional wisdom that places an obscure but pivotal group of organisms close to our own ancestry.

BRACHIOPODS were once called lamp shells, because they look like the lamps seen in productions of Aladdin. Like Aladdin's magic lamp, brachiopods have a genie inside. It is a feeding structure called a lophophore, a horseshoe-shaped arrangement of ciliated tentacles surrounding the mouth. This organ marks brachiopods as different from bivalve molluscs, which they resemble only superficially. But lophophores are also found in bryozoans, and marine worms called phoronids. Tradition has grouped these three phyla together on the basis of the shared presence of the lophophore, on the grounds that such a complex organ is unlikely to have evolved more than once.

Fitting the lophophorates into the larger scheme of things is more difficult. Animals more complex than flatworms conventionally belong to one of two large groups, the protostomes or the deuterostomes. Like Montagues and Capulets, the differences between the two are ontogenetic and hard to tell from adult appearance. In protostomes, the hole in the end of the blastula becomes the mouth, cleavage between the cells of the early embryo tends to be spiral, development is determinate, and the coelom (or body cavity) originates by the splitting of pre-existing mesodermal layers. Prominent protostomes include molluscs, arthropods and annelids: Drosophila is a protostome.

In deuterostomes, in contrast, the hole in the end of the blastula becomes the anus and the mouth develops separately (whence 'deuterostome', or 'second mouth'), cleavage is radial, development indeterminate, and the coelom develops at the same time as the mesoderm, from infolding regions of the primitive gut. The coelom in many deuterostomes is tripartite, arranged as three cavities from front to back. The second and third coeloms, if not always the first, are in turn subdivided into left and right halves. Deuterostomes include echinoderms and chordates: the best known is Homo sapiens.

Cleavage in lophophorates is generally thought to be radial, and the coelom is usually held to evolve from infoldings from the gut. The coelom also tends to be tripartite (although the presence of the foremost compartment is debatable), and the two halves of the lophophore originate from the two halves of the second pair of coelomic cavities. It is along these lines that lophophorates have been allied with the deuterostomes.
Embryological differences, though, are not always as clear-cut as these simple definitions imply. To complicate matters, lophophorates exhibit features that suggest a protostome affinity. Brachiopods, for example, have chitinous, hair-like structures similar to the setae of annelids.

If conventional wisdom has it that deuterostomes represent a monophyletic pearl amid a paraphyletic, protostome oyster, then the lophophorates can be placed as the nacre closest to the pearl, and yet not of it. Although protostomes, they occupy a special place as those closest to the ancestry of deuterostomes, having acquired features associated with deuterostome ontogeny, but not yet having lost primitive traits of protostomes.

And so it would stand, were it not for the unquiet stirrings of molecular evidence. Phylogenetic reconstruction based on ribosomal RNA gene sequences has allied brachiopods with molluscs. But the evidence gathered hitherto has been tentative and easily dismissed as more protosome primitiveness.

Perhaps no longer. Complete $18 \mathrm{~S}$ ribosomal RNA genes are now available for many species. Kenneth M. Halanych and colleagues use them to show that lophophorates are grouped firmly within the protostomes (Science 267, 1641-1643; 1995). Several different methods of reconstruction reach essentially the same result. Lophophorates are not just protostomes but advanced protostomes, and have no special relationship with deuterostomes.

In the consensus tree presented by Halanych et al., protostomes emerge as a strongly supported clade, but the deuterostomes (in this analysis a crinoid, an amphioxus and a lamprey) group together far less well. Within the protostome group, the arthropods branch off first and (now, this is the interesting part) the bryozoans come off next.

This leaves a 'crown' group of brachiopods, phoronids, molluses and annelids. Within this crown, no species is closer to any other, except that phoronids emerge as closer to articulate brachiopods (in which the shells are hinged together) than inarticulate ones (in which they are not).

In general, the result has fascinating implications. First, the presence of the lophophore defines a subgroup of the protostomes (which the authors call the Lophotrochozoa) that includes the lophophorates, molluscs and annelids, but excludes arthropods.

Second, the position of bryozoans, which are lophophorates and yet primitive with respect to annelids, molluscs and the other lophophorates, suggests that annelids and molluscs had a lophophorate ancestry, but their affinities have been masked by the secondary loss of this definitive structure.

Critics can hardly balk at the suggestion that annelids and molluscs are lophophorates that happen to have lost their lophophores, for the traditional alignment of lophophorates with deuterostomes requires that chordates and echinoderms should have suffered the same loss.

Nevertheless, one is entitled to wonder whether Halanych et al. are justified in throwing out much ontogenetic and morphological evidence on the basis of the sequence of a single molecule: particularly as there is another group of lophophorebearing animals, the pterobranchs, which do not feature in their analysis.

Like the lophophorates, pterobranchs are small, obscure sea creatures. Only three genera are known, but all have lophophores derived from the second pair of coelomic cavities. Yet zoologists do not group pterobranchs with lophophorates, but with the acorn-worms or enteropneusts, which lack lophophores. Pterobranchs and enteropneusts together comprise the hemichordates, and are deuterostomes. If the lophophorates are protostomes, as Halanych et al. suggest, then the lophophore must have evolved twice independently. The extent to which molecular evidence from hemichordates would dent the monophyly of the newmade Lophotrochozoa remains to be seen. And there is one further piece of evidence to be considered. It comes (one will no longer be surprised to learn) from yet another group of obscure sea creatures, this time one with which even zoologists are unlikely to have made much acquaintance. From the abyssal depths of the bathyal ooze has emerged a variety of acorn-worm of unusually piebald character. Although they look like the acornworms from inshore waters, they sport lophophore-like fringes of tentacles growing from a region that corresponds with the second pair of coelomic compartments. These animals are almost certainly deuterostomes, yet a lophophorate by any other name would smell as sweet. Whether the same applies to the Lophotrochozoa is less certain.

Henry Gee 